\title{
COMMEMORATIVE LECTURE Structural Insights into the Functions of the Large Ribosomal Subunit, a Major Antibiotic Target
}

\author{
Thomas A. Steitz \\ Investigator, Howard Hughes Medical Institute and Sterling Professor of Molecular Biophysics and Biochemistry, \\ Yale University Professor of Chemistry, Yale University, New Haven, CT, USA
}

(Received for publication on Ausust 25, 2007)

Key words: Ribosome structure, RNA, antibiotics, protein synthesis, ribozyme

\begin{abstract}
Chairman (Prof. Yoshikazu Nakamura, Institute for Medical Science, University of Tokyo): Finally we will have a lecture by Prof. Thomas A. Steitz. He is the winner of Keio Medical Science Prize. Already, he explained or talked about his personal history and therefore really I do not need to repeat the same thing; but briefly I would like to introduce his career. He graduated in Chemistry from Lawrence College, Wisconsin in 1962, and he finished his $\mathrm{PhD}$ in biochemistry and molecular biology at Harvard University. Then he started his professional career path in Harvard University, the same department of chemistry, and he also spent three years 1967-1970 in Cambridge, England, where he met so many important figures as we learned yesterday. Then he got an appointment in Yale University in molecular biophysics and biochemistry in 1970. Since then he has stayed in the same department, and carried up through an assistant professorship, associate professorship and full professorship. $\mathrm{He}$ has received quite many awards. Among those just I will mention a few important points. In 1990, he became a member of the National Academy of Sciences in the United States, and also the same year a member of the American Academy of Arts and Sciences. He received several awards: for example, in the year 2001, the Rosenstein Award for Distinguished Work in Basic Medical Science, and the latest one in the year 2004, the Frank Westheimer Medal of Harvard University. As you know, this year he received the Keio Medical Science Prize. He received it; I trust and I hope that this award is the best and prestigious award. Now we will ask Tom for this lecture about "Structural insights into the functions of the large ribosomal subunit, a major antibiotic target."
\end{abstract}

Prof. Thomas A. Steitz, Department of Molecular Biophysics and Biochemistry and Department of Chemistry, Yale University, and Howard Hughes Medical Institute: It is a great pleasure to be here, and thank you so much for that kind introduction. As I said yesterday, it is always wonderful to come to Japan and visit. My son and I had a great breakfast this morning; a sushi breakfast at the fish dock, ${ }^{* 1}$ the best sushi that I ever had in my life; it is going to be hard to do this again, I have to say, back home. My son is out having a good time in Tokyo and I am here having to talk, so I am going to have a good time too.

I would like to start out by giving an introduction to the ribosome and the ribosome problem, and then provide a few details about how we solved the structure of the large ribosomal subunit. Now, I realize that this is an unspecialized audience with respect to structure determination, with one or two exceptions obviously here, but I want to give a sense of what the problem was that needed to be solved and how we went about it, but that will only be five slides, so not to worry. Then I shall talk about what we have done to understand the mechanism of peptide bond formation. Finally, I shall talk about the antibiotic binding studies that we have done and the examination of the effect of resistance mutations, and finally talk about what Rib-X Pharmaceuticals, Inc.- the company that I and some of my colleagues started five years ago - are doing to use this information to design new antibiotics, which looks like it is going to be successful.

I shall start with a fact published in Nature a couple of years ago, that in the United States, two million people will acquire antibiotic resistance infections in US hospitals, and 90,000 will die of it. Indeed, this is a significant

Dr. Steitz is the recepient of the 2006 Keio Medical Science Prize. Presented at the Keio Medical Science Prize Symposium, November 2, 2006.

Reprint requests to: Dr. Tomas A. Steitz 266 Whitney Avenue, Bass 418 P.O. Box 208114 New Haven, CT USA 06520-8114

*1 A fish dock at Tsukiji in Tokyo. 
problem. Another important point is that the ribosome is a major target of antibiotics - in fact, the major targetand the large ribosomal subunit is the major target in protein synthesis. Consequently, our understanding of how a large ribosomal subunit works and how it is inhibited by antibiotics should indeed to be helpful to attacking this problem.

But let me back up and say a few things about our approach. There are some differences of opinions about how one should do structural biology. This is my opinion, which was exhibited very well by Dr. Yokoyama a couple of lectures ago. That is: you need to have the structures of every stage of a process in order to understand that process. It is not just good enough to have one structure; you need to have them all. So it certainly is not enough to look at the pieces. As you know, most assemblies are made from a lot of pieces. It is easy for us to be reductionists; we all reductionists, and we are tempted to look at whatever we can get our hands on, but knowing the structures of the pieces of a clock does not tell you how a clock works. But I might say that even if you know the structure of a clock sitting there, that does not tell you how a clock works either. So what you have to do is have the structures of the clock or the assembly that works at each step in that process. Even though some of the steps are metastable, you have to be a clever biochemist and figure out how to stabilize that state so you can take a snapshot of it, and then you can put it together into a movie. So that is the philosophy that we have taken with all the problems that we were studying in the lab. I did show some movies yesterday, and that is how we are looking at the ribosome as well.

We have been looking at all aspects of gene expression, but of course today I am just going to focus on the ribosome and indeed I am only going to focus on the large ribosomal subunit where peptide bond formation occurs. This schematic drawing of protein synthesis by Jim Watsonshows where our understanding of the ribosome was in 1964. It was known that the decoding happens in the small subunit and peptide bond formation occurs on the large subunit. Also shown was the A site, where the aminoacyl-tRNA binds, and the P site, where the peptidyltRNA binds, which he proposed existed, but he did not know about the E site, where the exiting deacylated tRNA binds, and he did not know about the polypeptide exit tunnel, but he did propose translocation. So we will mention a few things about the tunnel, and we will also mention about the E site.

The ribosome as I am sure you all know is the largest RNA assembly that exists. The eukaryotic ribosome is a bit larger than the prokaryotic ribosome, but otherwise there are not any larger RNA assemblies. The bacterial ribosome has 3,000 nucleotides in the large subunit, and another 1,500 in the small subunits; so the whole assembly of the $70 \mathrm{~S}$ is about two and a half million molecular weight, two-thirds RNA by mass. Think of it as an RNA machine contaminated with proteins, whereas the spliceosome, I tell my wife, ${ }^{* 2}$ is a protein machine contaminated with RNA. The ribosomes constitute one-fourth of the bacterial cell mass, so it is very abundant, and of course that is extremely useful if you want to do structural studies, to have a very abundant machine.

Now the early structural work in the mid-70's was done by Jim Lake using negative staining in electron microscopy, and he was able only to show the shapes of the two subunits, but his shapes for the individual subunits are largely right at this resolution of $50 \AA$ or so; then examining the model of the whole ribosome you can see that the two subunits are sort of snuggling up here like a pair with the small subunit lying on top of the large subunit. Using cryo-electron microscopy (cryo-EM), it was possible to go to somewhat higher resolution by 1997 and position these transfer RNA (tRNA) molecules approximately correctly, showing the CCA going into the large subunit and the anticodon loops of decoding a fictionally placed messenger RNA, and then the E-site tRNA, and more or less over here but again not exactly positioned correctly. So that is where we were in our understanding of ribosome structures approximately 10 years ago.

I have met up with Venki Ramakrishnan many timesactually, he was a post-doc at Yale in the 1980s with Don Engleman and Peter Moore-but I met him in Sweden at a meeting, so we had a photo opportunity, and I am going to use Venki's structure of the small ribosomal subunit and put it together with our structure of the larger ribosomal subunit to get the structure of the whole $70 \mathrm{~S}$ ribosome. The two subunits were positioned using the coordinates of the phosphate backbone of the 70S from Harry Noller and his three A-site, P-site, E-site tRNAs. Venki, as you may have noticed if you are paying attention to this area, has recently determined at 2.8 angstroms resolution the structure of the $70 \mathrm{~S}$ assembly with tRNAs bound in the P site and E site and partially the A site (5); so there is an even more accurate structure of this assembly than shown in Figure 1 but I think for our present purpose you would not be able to tell the difference, nor would I. The ribosomal RNA is shown in white and purple for the large subunit and in various colors of yellow for the small subunit. At the bottom of a large cleft in the 50S subunit is where we are going to spend a lot of time, actually, where the peptide bond formation occurs. There is a hole in the small subunit where the mRNA comes in. You can see a very complex folding of the RNA. Some gaps in between, for sure, but many of these gaps are filled by the proteins that are fitting in between.

*2 Prof. Joan A. Steitz, Department of Molecular Biophysics and Biochemistry, Yale University, and Howard Hughes Medical Institute. 


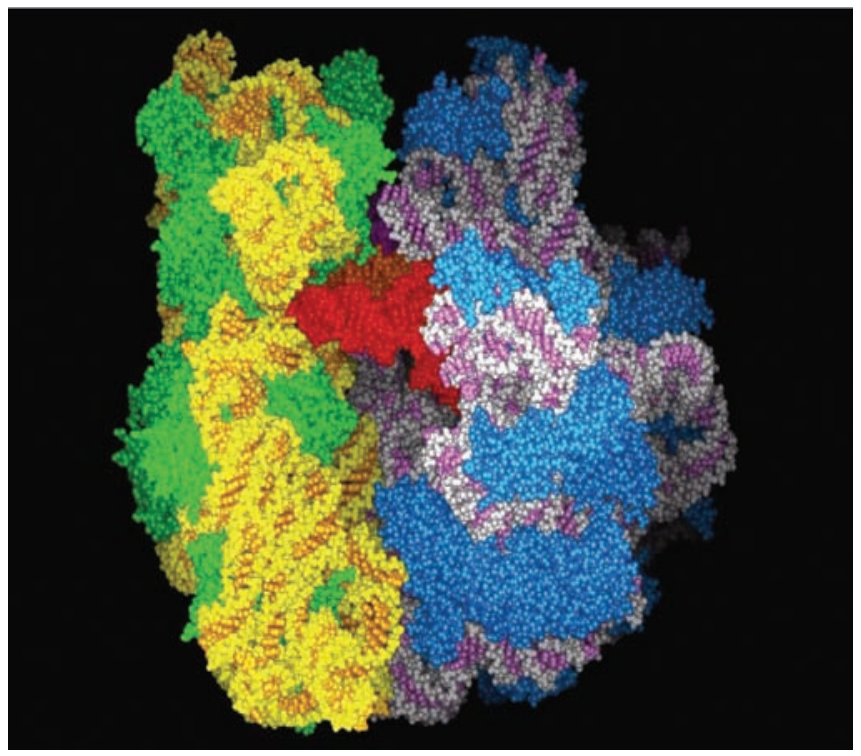

Fig. 1 Space-filling model of the 70S ribosome derived from the structures of the Hma large subunit (Ban et al., 2000) and the T. thermophilus small subunit (Wimberly et al, 2000) docked using the rRNA from the model (Yusupov et al., 2001) of the 70S ribosome, and that includes the A-, P-, and E-site tRNAs from that model also. The 23S rRNA and 5S rRNA are on the right, whereas the $16 \mathrm{~S}$ rRNA is on the left. rProteins are shown. The A-site tRNA and the P-site tRNA are shown with their 3' ends extending into the peptidyl transferase cavity. (Reproduced from "RNA in the Synthesis of protein IN: The RNA World." PB Moore, TA Steitz, Cold Spring Harbor Laboratory Press, 2005; 258 Fig.1)

Well, what I would like to first do is use a movie that Venki Ramakrishnan commissioned to show the overall process of protein synthesis using both crystal structures that are known and electron microscopy reconstructions. Ultimately I want to say that what we need to have are the high resolution crystal structures for every one of these steps. For the moment we are doing pretty well because we have a lot of electron microscopy images of these steps which combined with crystallography provides a pretty good idea of what is happening, but I think we shall want to get down to the exact chemistry. Thus, we are going to have to have crystal structures., but this is where we are at the moment. This movie comes from the Medical Research Council (MRC) laboratory. First, the small subunit binds to the messenger RNA and is facilitated by initiation factors, IF2, IF1 and IF3, and they then position the formyl-methionine-tRNA (fMet), the initiator tRNA. Then that assembly meets up with the large subunit, and the factors dissociate leaving the fMettRNA positioned in the $\mathrm{P}$ site. Now elongation begins with the delivery by EF-Tu of an aminoacyl-tRNA, GTP gets hydrolyzed and then you get a tRNA accommodation. There will be a little "zing" here as the peptide bond is formed; then it will go to what is called the hybrid state, which moves the CCA ends over one step into the E site and P site. Now EF-G comes along, hydrolyzes GTP, translocates both tRNAs and then another tRNA is delivered by EF-Tu, and this process goes on at a faster rate in the movie. You see now polypeptide coming out the tunnel; there is a tunnel that goes about 100 angstroms through the middle of the large subunit, and the folding of the polypeptide happens on the other side of the tunnel. Actually, another interesting process is the cotranslational insertion of polypeptides into the membranes which we do not know anything about yet in detail. Then the factor comes along to stimulate the hydrolysis of the peptidyl-tRNA which then releases the protein, and now a couple of proteins come along to clean up the job of removing the message and dissociating the complexes. So that is the whole process, and now we are going to focus our attention on the large subunit.

I should mention that my work on the ribosome was initiated when Nenad Ban joined my laboratory in 1995. I had certainly considered initiating this project for a long time; Ada Yonath's lab had been pursueing the ribosome for a few decades and many of us noted that while she had been successful at growing some crystals, the success at solving the structure had not been so noteworthy. So we thought we should have a hand at it as well, and I thought Nenad Ban was the right guy, and indeed he was. He came in and he grew the crystals repeating the crystallization, I might say, of Ada Yonath, and then made heavy atom derivatives of the critical kind. I am going to come back to what he did that was so good. Then he was joined two years later by Poul Nissen. Poul Nissen came from Aarhus and he has gone back to Aarhus; he figured out how to make fatter crystals with fewer of the kinds of problems that the earlier crystals had. He worked with Nenad Ban to solve the structure. So really the structure was solved by these two wonderful post-docs, with occasional help from a few other rotating graduate students and another postdoc in the lab.

What were the crystallographic problems? Here are my five slides, but I want to give you an indication of why it was that it was difficult to solve the ribosome. I must say that from the bottom of Mt. Everest, which is what the ribosome structure determination was considered, its structure determination looked very daunting. Now of course, every time you get to the top of the mountain, you say, "Well, gee, that was not such a bad thing, It really looks pretty easy." But at the bottom, it looked bad, and why was that? Well, the crystals initially were kind of thin, they had a number of problems and they were twinned. We could get very good diffraction, but Poul Nissen figured our how to make fat, well-behaved crystals. So that was a great start. The other problem, in fact the major problem-solving novel protein structures from the days of myoglobin on, is making what is called a 


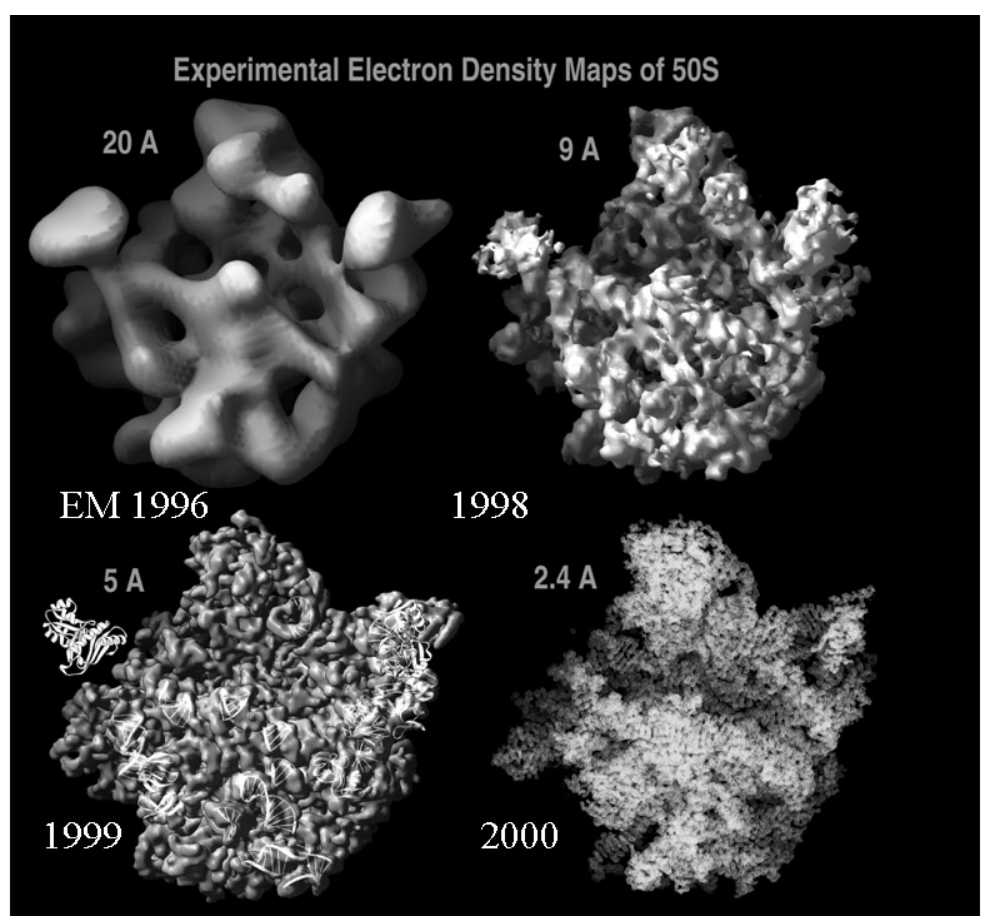

Fig. 2 The appearance of the large ribosomal subunit from $H$. marismortui in electron density maps at different resolutions. The subunit is shown in the crown view at (a) $20 \AA$ resolution, (b) $9 \AA$ resolution (Ban et al., 1998), (c) $5 \AA$ resolution (Ban et al., 1999), (d) $2.4 \AA$ resolution (Ban et al., 2000). CP designates the central protuberance. The L1 stalk, which is visible at low resolution, disappears as resolution improves.

heavy atom derivative of the crystalline complex and locating that heavy atom. How do you do that in this case? It is a very large assembly; it is one and a half million molecular weight, and if you add just a single uranium atom, even though you think of uranium as being big, it is very, very small compared to the ribosome. So what was done was to use a very large heavy atom cluster compound. The compound, containing 18 tungstens plus a few other atoms, has 2,000 electrons, notably larger than uranium. Now why is the cluster compound useful? It is useful because at low resolution, that is, at a small diffraction angle, not the angle that is going to tell you where all the atoms in the molecule are, this cluster scatters as one assembly. You get the square of the number of electrons as your signal. Squaring 2,000 rather than 76 is a big thing. So if you look at the intensity of the scatter as a function of resolution: at $12.5 \AA$ resolution, which is almost electron microscopy resolution, you know, low resolution-and look what is happening to the scatter for these cluster compounds, the intensity of the scatter goes through the roof. That means we have a very strong signal from the heavy atom cluster compound. Thus, we calculated the first maps at $12 \AA$ resolution. Now, most of you will not know what a difference Patterson map is and I will not try to tell you what it is, but it is what we look at to locate the positions of heavy at- oms, and we have to see a peak. You can recognize a peak, which there is, and these peaks in different projections have to be correlated in a particular way, which they are. So when Nenad Ban calculated this, we could all say "Eureka! We know this is going to work." Indeed, it did.

Figure 2 shows the progress in structural studies of the large ribosomal subunit. In 1996 there was electron microscopy at $20 \AA$ resolution. I always say this E.M. image looks like a Henry Moore statue here; it looks like a man on his knees holding some sort of a torch with his head here. In any case, there are a lot of holes in it. In 1998, we could see in the first $9 \AA$ resolution x-ray map what looks like RNA helices, so this was the first time that one could see recognizable structural features in an electron-density map of the ribosome. Now in 1998, we published how we were doing business, ${ }^{1}$ and then a whole lot of other groups started doing exactly the same thing, quite reasonably, and by 1999 , there were several simultaneous or almost simultaneous publications of $5 \AA, 6 \AA$ or $7 \AA$ resolution maps and at that resolution we could fit in little pieces of known ribosomal protein structures, which was nice. In 2000 we got a $2.4 \AA$ resolution map and we could fit all 100,000 atoms. So then we knew the atomic structure of the large ribosomal subunit. $^{2}$ 
This representation of the structure of the 50S subunit that was published in $2000,{ }^{2}$ shows the RNA backbone in orange and bases in white fairly tightly packed around the active center and the proteins embedded around the surface (Fig. 3). I am not going to go in to detailed discussion of what we learned about the structure, but there was a lot that we were able to understand about principles of RNA folding and RNA tertiary structure interactions and how in the world a large molecular assembly is made out of RNA. But I will not talk about that today. What we are going to do is we are going to split the subunit in half so we can look in the inside; splitting it like an apple and opening it up. In white are those RNA regions that are in the interior that has been split and in green the protein penetrating into the interior helping to pack the interior and hold it together. There is the peptidyl transferase center where we are going to spend most of the rest of the time, and there is that exit tunnel, through which the newly synthesized polypeptide goes and then folds outside the far end; it is about $20 \AA$ in diameter in some places and it is $10 \AA$ in others and it is about $100 \AA$ long. All the antibiotics we are going to talk about, by the way, bind in the tunnel either in the region below the peptidyl transferase center or a little bit closer to the site of the peptide bond formation.

The active site is composed entirely of tightly-packed RNA, and I might say is very conserved. Fortunately, it is not $100 \%$ conserved, otherwise antibiotics would not work as therapeutics, but it is very conserved. Shown in red are all the rRNA nucleotides that are conserved, very conserved, and if you consider that eubacteria, archaebacteria, eukaryote...We are talking about all of evolution; that is, the conservation is very high. There is where the substrate binds. If we ask "At the point where peptide bond formation occurs, what is the closest protein?", the answer in 2000 in the Hma 50S subunit was "nothing very close at all"; now one can see in a eubacterial species, there is a protein, L27, that comes a little closer, but not much. So we were able to conclude that ribosome is a ribozyme; that had been speculated over the years because how else could you have a machine to make a protein if you did not have a protein? It is the chicken and the egg problem; which came first? The answer is: RNA.

What I would like to turn my attention to now, however, is how can we understand how an RNA is able to catalyze the chemical reaction of peptide bond synthesis? What is it about the RNA that allows that to happen? This is the work primarily of Martin Schmeing, however, I might say, Jeff Hanson helped a bit in the early stages. So here is a picture of Martin, the graduate student-or was; he is now a post-doc with Venki Ramakrishnan helping Venki conquer the world; Martin is very goodand he is shown next to his sailboat that is parked on our beach in Connecticut; I tried to get him to stay a little bit longer, so I said "You could keep your boat on our

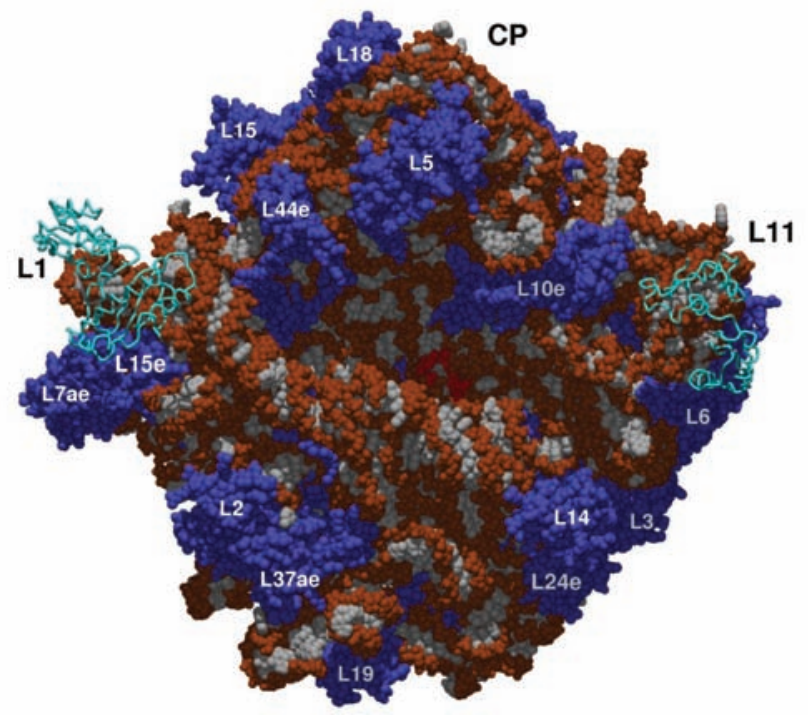

Fig. 3 A space-filling model of the large ribosomal subunit from H. marismortui with a transition state analog bound viewed down the active site cleft (Nissen et al., 2000). Bases are white, the sugar-phosphate backbone is grey and the substrate analog (in the center) is dark. Proteins whose structures are defined by the $2.4 \AA$ resolution map are black. Grey ribbon represents proteins L1 and L11, whose structures are independently known that have been positioned approximately using lower resolution electron density maps. Identification numbers are provided for all proteins, and CP designates the central protuberance. (Reprodced from "The Structural basis of ribosome activity in peptide bond synthesis." Nissen et al., Science 2000; 289: 920 Fig.4)

beach," and then winter came and he went off to Venki. Martin is a wonderful person, and as I mentioned yesterday, his mother was a roommate of my wife's and I was best man at his father's wedding, and I did not meet him until he walked into my laboratory. It was wonderful.

What is the reaction that is catalyzed? You are all taught this in introductory biochemistry. The alpha amino group of the aminoacyl-tRNA attacks the carbonyl carbon of the peptidyl-tRNA. It forms an oxyanion containing tetrahedral carbon intermediate, and then it breaks down to give products with the peptide being transferred to the A-site tRNA.

We are always optimists, so we of course did try to diffuse aminoacyl-tRNA into our crystals, even though we knew this was lunacy and it would not work, and of course it did not. So what we actually have done is we have used small substrate analogues-CCA-amino acid or CA-amino acid or CCA-peptide. We have looked at various combinations of all these complex structures. We have many analogues of the transition states that we have looked at and also of structures of the products. I will show you a few of these structures, but I am not going to show you anything like the large number that we have determined. Again, if we cut the ribosome in half 


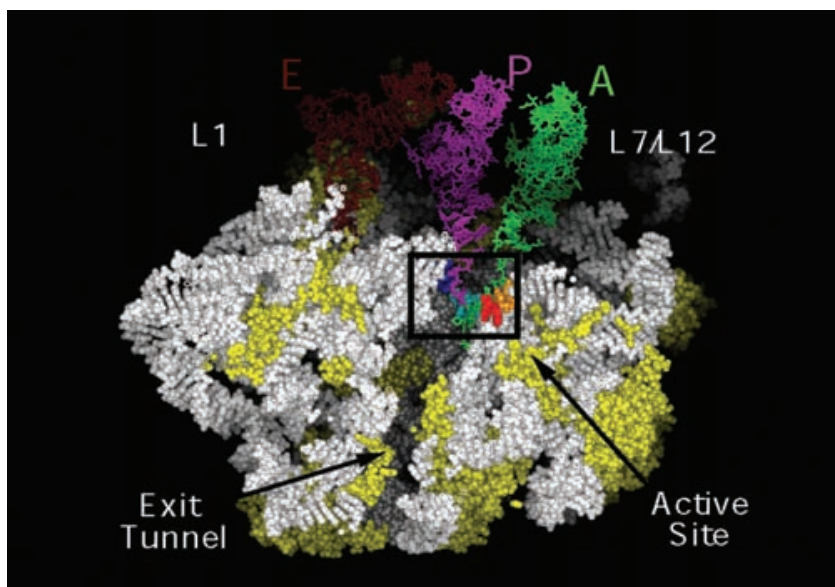

Fig. 4 The structure of the large ribosomal subunit from $H$. marismortui with products of the fragment reaction (CCA and C-pmn-pcb) bound in the peptidyl transferase center. A space-filling representation of the complex with three intact tRNAs added in the positions tRNAs assume when bound to the $\mathrm{A}, \mathrm{P}$, and $\mathrm{E}$ sites of the $70 \mathrm{~S}$ ribosome (Schmeing et al., 2002). TRNA is white, and the ribosomal proteins are grey. The subunit, which is oriented in the crown view, has been cut in half along a plane that passes through the peptide exit tunnel, and the front of the structure has been removed to exposed the tunnel lumen. The active site area is in a box. A close-up of the active site showing the peptidyl product (CC-pmn-pcb) bound to the A-loop. (Reproduced from "A pre-translocational intermediate in protein synthesis observed in crystals of enlymatically active 50S subunits." Schmeng et al., Nature Structural \& Molecular Biology 2002; 9: 225-230 Fig.4a)

so that we expose the polypeptide exit tunnel-you can see the proteins that come in and contact the tunnel (Figure 4). Here are the positions of the three tRNAs and this is where we are going to look at the substrate analogues that we have found. As I often point out, for the rest of the lecture, even though this is, I think pretty good research, we are going to think inside the box that surrounds the PTC rather than outside the box.

This is where we were in our understanding of substrate binding to the $\mathrm{PTC}^{3}$ slightly after 2000 . We knew from Harry Noller's work approximately where the two tRNAs are positioned, and of course the two tRNAs are oriented parallel to each other, but their $\mathrm{CCA}^{* 3}$ ends are rotated relative to each other by 180 degrees. This is the CCA of the P-site substrate, and C74 and C75 are making two base pairs with the ribosomal $\mathrm{P}$ loop, and the A-site substrate makes one base pair with the A loop. It is rotated by 180 degrees, so that the alpha amino group of the A side substrate can be close to the carbonyl carbon of the P-site substrate. Now, we thought this looked pretty good, sort of, except that we realized that this alpha amino group was not positioned quite right for the nucleophilic attack, but we sort of ignored and said, "Well, maybe it is accuracy," but it actually had something to do with the way we did our experiment.

Well, I went to Sweden a couple of years ago, and after my lecture, somebody asked this question: why is the peptidyl-tRNA not hydrolyzed in the absence of the A-site substrate? Now, since the rate-limiting step in peptide bond formation is the delivery of the aminoacyltRNA, the A site is mostly empty. If the enzyme is set up with the correct structure to catalyze the nucleophilic attack, why can it not activate water molecules, which actually it does in the final step of deacylation of the polypeptide? Well, I did not know what the answer, so I said, "I do not know what the answer is." I went back, and Martin Schmeing had already worked it out while I was having fun in Sweden. It turns out that the answer is that when the correct A-site substrate binds, it rearranges the structure of the active site in such a way that now the ester-linked peptidyl group gets correctly oriented and gets exposed. ${ }^{4}$ It is buried in the absence of a correct A site substrate.

If you look at the position of the peptidyl group, when you have just the peptidyl- CCA bound, and nothing in the A site, this peptidyl group is protected on one side by a $U$ base, at the position that a water molecule will have to take in order to attack. It cannot get there because it is sterically excluded. Similarly, on this side it is sterically excluded by some other RNA. So the enzyme ribosome is very clever. It is protecting its substrate in the absence of the A site substrate.

This is a complicated slide (Figure 5), but I will show you a movie shortly, which I think will clarify any questions you might have. What we were able to do, or what Martin Schmeing was able to do is look at one substrate that does not induce the change, and that is shown on this lighter color here, and the bases of the ribosome are shown also in lighter color. When that shorter substrate is bound, or in the absence of this substrate at all, this $U$, as I had shown in the previous slide, is maintaining the position of the peptide in this orientation and burying it and excluding water. However, if you add CCA, or if you have an intermediate complex which is shown in black, CCA, there is a conformational change in the ribosome, which is interesting and finally results in the moving of this base out of the way so that this peptide can reorient and you can get a nucleophilic attack. It is reoriented for the attack by the alpha amino group. Let me show a movie of the induced conformational change.

So we start out with the peptidyl group protected: you see this U base moving out of the way. The PTC is going to the induced structure. Now the alpha amino is in a position so that it can attack, which it does, and then you get the intermediate. That is what happens. Now this is

*3 The universal CCA trinucleotide common to all tRNAs located at their 3'-ends is shown to play an important role in peptidyl transfer reaction. 


\section{Proper binding of an A-site substrate induces conformational changes that exposes the ester of the P-site substrate for atttack}

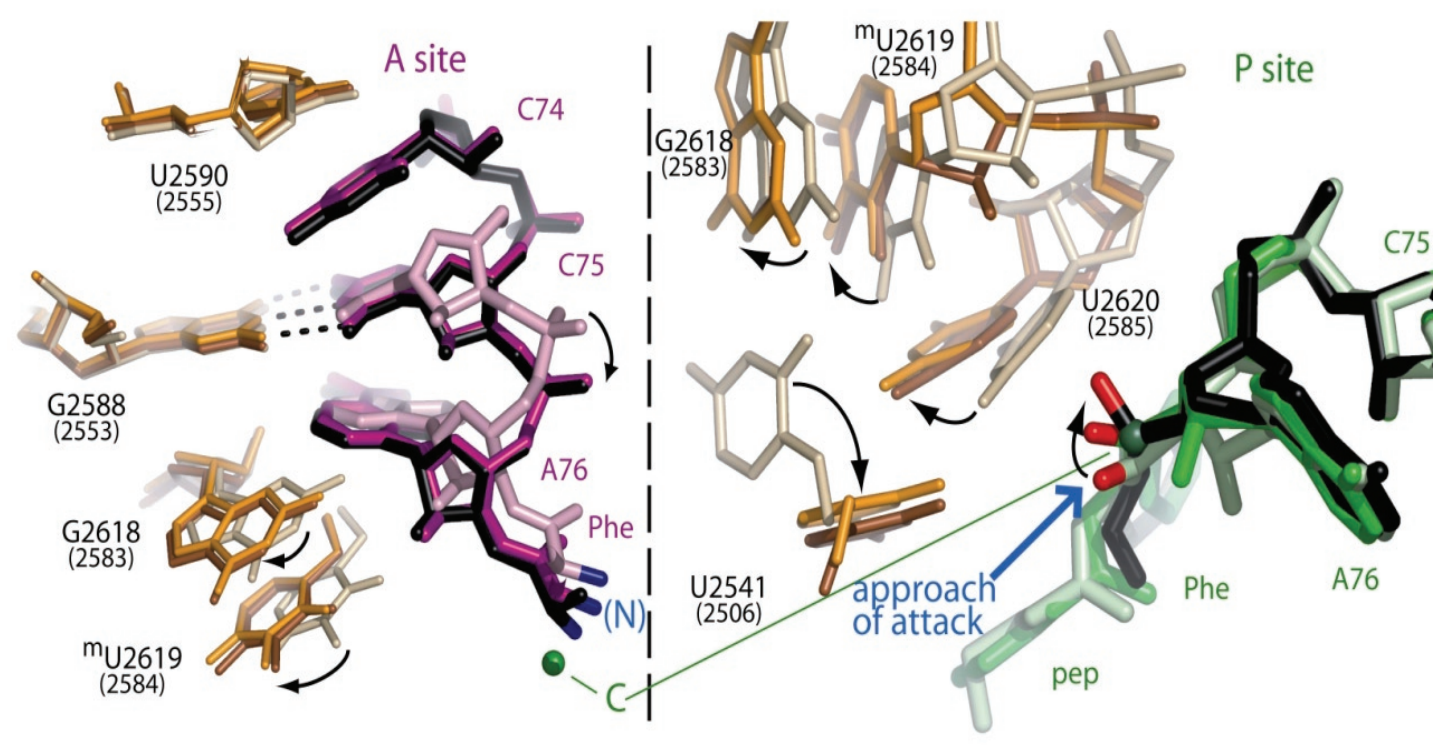

Complex 1: ChPmn + CCApcb, RNA in beige

Complex 2: CChPmn + CCApcb, RNA in orange

Complex 3: Transition state analogue (DCA) in black,

Fig. 5 Movements of rRNA and peptidyl-rTNA are induced by proper binding of the A-site substrate (Schmeing et al., 2005a). (a) A view of the A-site with three different substrate analogs bound. Without C74, ChPmh and rRNA colored lighter is positioned higher in the A-site. In substrates with C74, both CChPmn (darker rRNA) and the transition state analogue (TSA ) (black rRNA), C74 stacks with rRNA base U2590 (2555), shifting the substrates down and the $\alpha$-amino group closer to the ester carbon of the P-site substrate, rRNA base G2618 (2583) shifts to maintain the A-minor interaction, causing methylU2619 (2584) to also move. (b) View of the P-site of the same three complexes. The movement induced in G2619 (2583) by A-site substrate binding breaks its G-U wobble pair with U2451 (2506), which swings 90 . MethylU2619 (2584) and U2620 (2585) also shift to allow the ester group to move from the position it occupies when CCApcb (light) is bound together with ChPmn, to that when CCApcb (medium) is bound with CChPmn, and finally to that when it has been attacked by the A-site substrate, as shown with the TSA. (Reproduced from "An Induced-fit mechanism to promote peptide bond formation and exclude hydrolysis of peptidyl-tRNA." Schmeing et al., Nature 2005; 438: 520-524 Fig.3)

the proper structure for a pre-reaction ground state (Figure 6). So this is the cast of characters and where they are when chemistry is about to happen. So what is happening? There is the attacking alpha amino group, and it is interacting with the 2' hydroxyl of the P side substrate, and it also interacting with A 2451, A2486 in the Haloarcula marismortui numbering.

Well, why is that useful for catalysis of peptide bond formation? The first thing that any enzyme will do is to correctly orient the substrates, so they are ready for attack, and that is probably the biggest energetic factor to anything in catalysis. Jenks said this in 1971: entropy or loss of entropy by orienting the substrate is the biggest factor in catalysis. So that is important.

But what else is happening? Are these functional groups activating that alpha amino group? Well, we had suggested that maybe this A was doing something, but that was soundly shown to be incorrect, most effectively by Rachel Green, ${ }^{5}$ who showed that if you used the full tRNA in the A side, you can mutate that A to any of the three other bases, and it does not make any difference to peptide bond formation. Consequently, that $\mathrm{A}$ is not involved in catalysis. Well, there is only one thing left, and that is the 2' hydroxyl group of the P-site A76. Scott Strobel's lab showed ${ }^{6}$ that if you make that A76 sugar a 2' dioxy, and get rid of that P-site 2' hydroxyl, the rate of peptide bond formation goes down by one million fold; that is a big number. I will come back to this; I think that this large decrease is not entirely what the catalytic enhancement is relative to non-catalytic but it is a big effect.

Some earlier work by Andrea Barta in Vienna ${ }^{7}$ using a small peptidyl-AMP substrate has seen a similar but not such a large effect, and she proposed the following 


\section{The pre-reaction ground state}

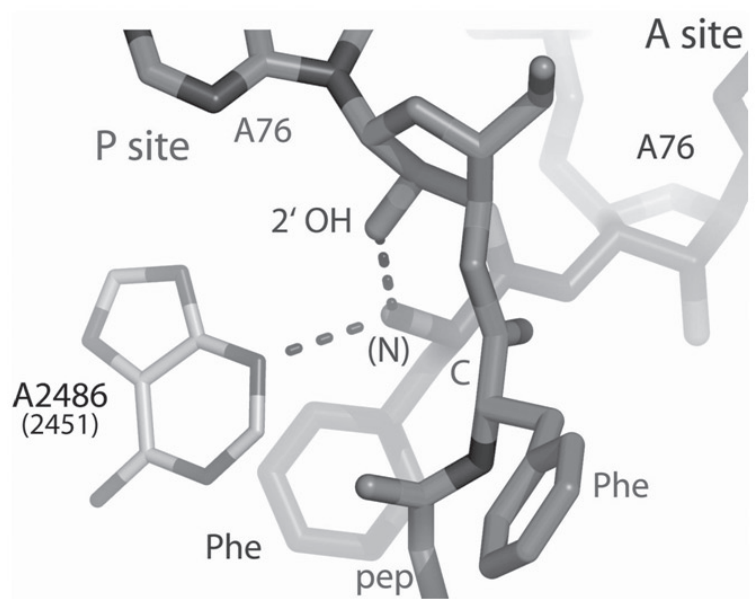

Fig. 6 Pre-attack conformation of the substrates of the peptidyl transferase reaction. The hydroxyl group representing the $\alpha$ -amino group of the A-site substrate, CCh-Pmn, is in position to attack the ester group of the P-site substrate CCApcp. It is within hydrogen-bonding distance of the N3 of A2486 (2451), and the 2'-hydroxyl group of the P-site substrate. In the ground state, the two reactive groups are $3.7 \AA$ apart (Schmeing et al., 2005a). (Reproduced from "An induced-fit mechanism to promote peptide bond formation and exclude hydrolysis of peptidyl-tRNA." Scheming et al., Nature 2005; 438: 520-524 Fig.4)

mechanism. Why is this two 2' hydroxyl so effective? Well, it picks up a proton from the attacking alpha amino group, and donates a proton to the leaving three 2' hydroxyl (Figure 7). It turns out that this P-site 2' hydroxyl is completely buried, so water cannot get there any other way. Anyhow, I think this proton shuttle has a big effect. Then we can ask, is the transition state being stabilized? That is the other thing that enzymes do. We have a nice complex, and we can see a water molecule which is positioned by the ribosome, ${ }^{8}$ and it could be the water molecule which is polarized is also contributing, but it is hard to estimate how much. So in the end we conclude that catalysis is accomplished by substrate orientation, the proton shuttle of the 2' hydroxyl and maybe some stabilization of the transition state by a water molecule.

This then summarizes how catalysis occurs at the PTC. I think we basically understand it at this point, and it fits with a lot of kinetic and modification data by others. So let me just wind up with a movie. Just to put this all together: this is a movie made by Martin Schmeing.

We are moving into the peptidyl transferase center. I can still talk over all this music. Here is the P loop and here is the A loop. That is the P-site substrate coming in, making these two C-G base pairs, and it is buried, the ester-linked peptide is protected. Here is the attacking A-site substrate coming in. It is making hydrogen bonds
A possible role for 2' $\mathrm{OH}$ on $\mathrm{A} 76$ of the P-site in chemical catalysis

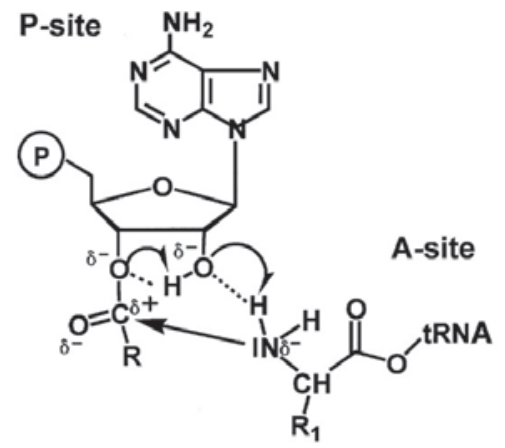

Fig. 7 A schematic drawing of the proposed "proton shuttle" model for peptide bond formation (Dorner et al., 2005). The P-site peptidyl-A76 is being attacked by the A-site aminoacly-tRNA. Protons are shown shuttling from the $\alpha$-amino group to the 2' $\mathrm{OH}$ and from the 2' $\mathrm{OH}$ to the 3 ' hydroxyl group. (Reproduced from "Molecular aspects of the ribosomal peptidyl transferase" Doner et al., Biochem Soc Trans 2002; 30: 1131-6 Fig.3)

with the A-loop being oriented, and you can see that it is not well oriented for attack, but now if we add another $\mathrm{C}$ to form CCA, you get the activated form. Now we are ready for the attack of the alpha amino group on the carbonyl carbon. Then we get the transition state. It is stabilized by the water molecule. Then it breaks down. Then we have the products. Now the deacylated CCA will then go to the E site, the exit site. Martin Schmeing determined the structure of an analogue of an E-site substrate - actually, the whole tRNA goes, of course, but we do not know where the rest of it is, for the moment. Anyhow, this lonely CCA is wandering over to a binding site that exists for the CCA. I might say this binding site is different between eukaryotes and prokaryotes and affects what kind of antibiotics will bind here. I am not going to talk about that. The reason that the amino-acylated tRNA cannot bind is because there is no room to accommodate the amino acid. Now as the peptidyl-CCA travels from the A-site to the P-site you can see this 180 degree rotation, going from the A site to the P site; the peptidyl CCA has to rotate 180 degrees, and now one is ready to start all over again. That is the reaction.

So now let me turn to the antibiotic studies for the last 15 minutes or so. These were done largely by Jeff Hanson initially. He started off by looking at erythromycin, but it did not bind. That is because it turns out that archaea are eukaryotes in disguise in many ways. That is, many of the binding sites for antibiotics in this Haloarcula marismortui look more like the mammalian binding sites than they look like the eubacterials. Consequently, 


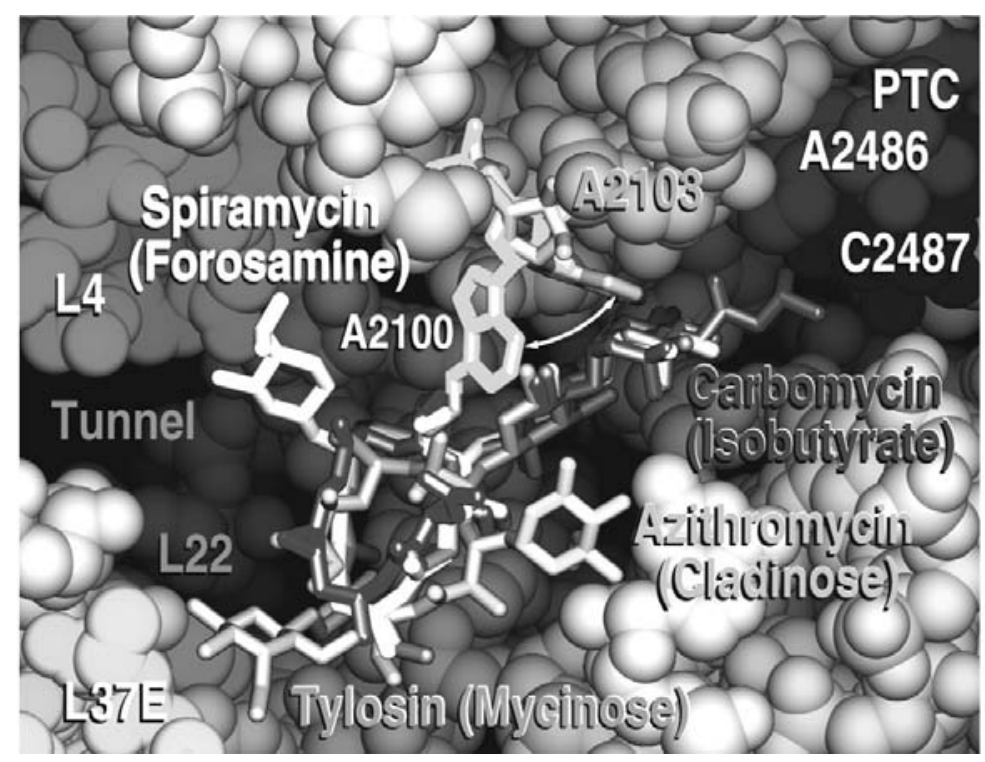

Fig. 8 The interaction of macrolide antibiotics with the large ribosomal subunit of H. marismortui (Hansen et al, 2002b). A superposition of several macrolides/large ribosomal subunit complex structures. Structures containing carbomycin, tylosin, spiramycin, and azithromycin have been superimposed by aligning corresponding 23S rRNA atoms in four independently determined crystal structures. The macrolide rings of the four antibiotics bind to virtually the same site in the proximal portion of the peptide exit tunnel. In the case of the 16-membered macrolides examined (tylosin, carbomycin, and spiramycin) A2103 (2062) swings down so that its N6 can form a covalent bond with their aldehyde substituents. (Reproduced from "The Structures of Four Macrolide Antibiotics Bound to the Large Ribosomal Subunit." Hansen et al., Molecular Cell 2002; 10: 117-128 Fig.5)

he could not make a complex with it. But he could look at other macrolides that bound more tightly. So for example, erythromycin, which was a 14-membered ring, did not bind; but azithromycin which is a drug made by Pfizer, selling for a billion and a half dollars or so a year, did bind, and tylosin, which has a 16-membered macrolide ring, did bind. Most of these others in the MLSK class did not bind, however. But the 15-and 16-membered macrolides did bind, and they bound in the tunnel. If we superimpose more or less where they all are, you can see that they are roughly binding the same place (Fig. 8); they each have different sugar substituents, and these sugars are reaching out and touching the bases. All of the interactions are with RNA. There is some little interaction here with L22, with tylosin, but otherwise all the interactions are with the RNA. Some of them have extensions that go up towards the peptide bond-forming regions, the peptidyl transferase center, and we saw a covalent link formed between these 16-membered macrolides and this $\mathrm{A}$.

How, then, do macrolides work to inhibit protein synthesis? Here is where they are located.The binding site for the macrolides is in this tunnel, and if we look up the tunnel here, this is what it looks like. There is the peptide bond-forming region. It is quite empty, looking up the tunnel, and then here is carbomycin bound partly blocking the tunnel. It is blocking the egress of the polypeptide. I call it molecular constipation; it just stops the polypeptide from coming out.

We wanted to look at the effect of mutations on the resistance. Since we have, so to speak, the resistant strain, that is we have the one that looks like the eukaryote, we wanted to go backwards and make it look like the eubacteria. What we have is a $\mathrm{G}$ in this position. If you mutate A2058 to a $\mathrm{G}$ in eubacterial ribosomes, that reduces the binding constant for erythromycin by 10,000 fold. So that is why it does not bind to our subunit since we have a $\mathrm{G}$ in this region.

All those antibiotics that I showed you on the previous slide of the MLSK antibiotic, mostly do not bind because this is a G. Why is that G a problem? Well, if you look at the one that does bind, the 16-membered macrolide, the ring is right above this two-amino group of the G. We posited that that is getting desolvated-energetically unfavorable-and the packing is not quite as tight, because the macrolide ring has to back off a little bit due to this amino group end poking into its center. So we thought, what if we changed this $\mathrm{G}$ back to an A? Consequently, Daqi $\mathrm{Tu}$, a joint graduate student of Peter Moore and myself, and Gregor Blaha, a post-doc in my lab, did a little genetics on Haloarcula marismortui; not a wonderful genetics system, I have to say. They mutated that $G$ to an A, and then said, "What happens?" Well, it turns out all the macrolides, lincosamides, streptogramins, and ketolides (MLSK) antibiotics bind just fine. ${ }^{9}$ So here they are. We were not able to bind clindomycin before, 


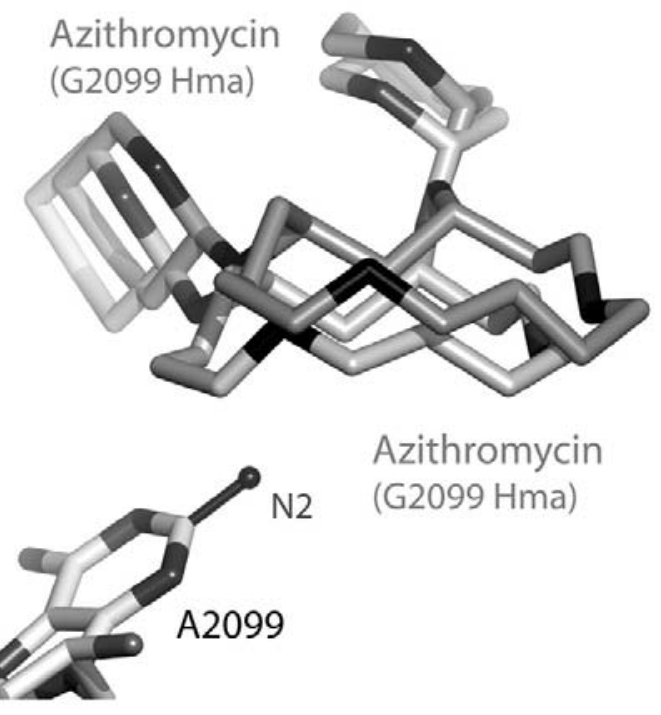

Fig. 9 Macrolide interactions with the large ribosomal subunit of H. marismortui. Comparison of the location and conformation of azithromycin bound to the wild-type $H$. marismortui large ribosomal subunits, and G2099A mutant large ribosomal subunits from the same organism (Tu et al., 2005). Structures of both forms of the subunit with the drug bound have been superimposed. (Reproduced from "Stractures of MLSBK antibiotics bound to mutated large ribosomal subunits provide a structural explanation for resistance." Tu et al., Cell 2005; 121 : 257-270 Fig.2F)

but it binds fine to the G2058A mutant ribosome; virginiamycin $\mathrm{S}$ and $\mathrm{M}$ bind, and so on, and erythromycin binds wonderfully. If you compare what we were able to do when we had the wild-type subunit in three millimolar G2058A mutant erythromycin, it did not bind. However, at 0.003 millimolar, when we have only $33 \%$-we have now made it $100 \%$ mutant subunit, but we could even do it with $33 \%$ of the crystal being this-it binds just fine. So we figured that we have changed the affinity by 10,000 -fold, that is with just that one change, we have completely reversed the affinity. What happens with azithromycin? We can bind azithromycin to wild-type and mutant Haloarcula marismortui large subunit; it binds almost exactly the same way as when it was bound to the mutant; it is just raised up maybe an angstrom or a fraction of an angstrom because of the presence or absence of this N2 group of the G. (Figure 9.)

This result says a couple of things about our antibiotic studies. One of the things it says is that our complexes with this Haloarcula marismortui 50S subunit are good for drug design, because even if the affinity is down, the way in which the substrate binds is largely the same. There has been some work on antibiotic complexes done by Ada Yonath, but I have taken all of those comparison slides out. I think most of her work that can be com-

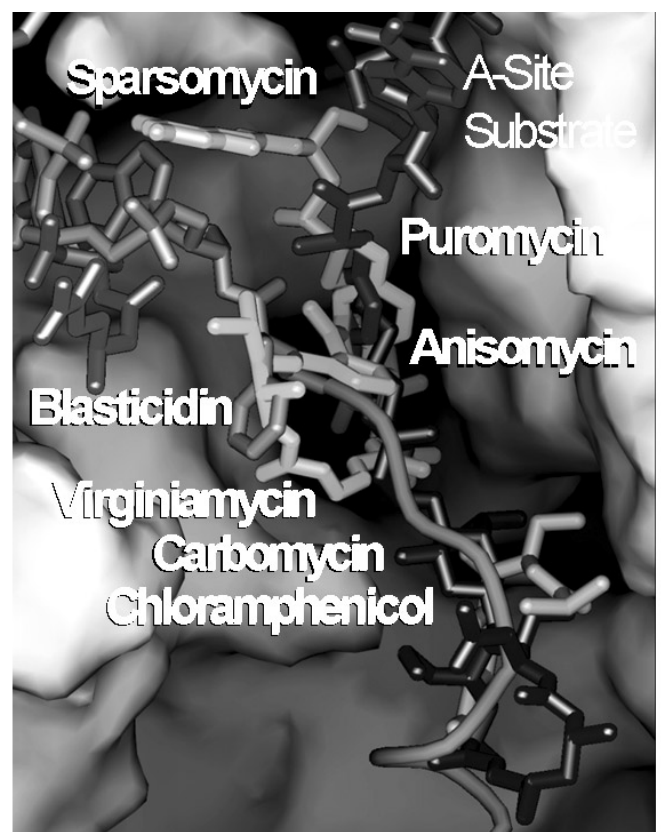

Fig. 10 The positions of seven antibiotics and A-site plus P-site substrates bound to the peptidyl transferase center (Hansen et al., 2003). The ribosome has been split open to reveal the lumen of the exit tunnel and adjacent regions of the peptidyl transferase site. Ribosomal components are depicted as a continuous surface at two positions where splayed-out bases provide hydrophobic binding sites for small molecules. Seven independently determined cocrystal structures have been aligned by superimposing the $23 \mathrm{~S}$ rRNA in each complex. (Reproduced from "RNA in the synthesis of protein IN: The RNA world." PB Moore, TA Steitz, Cold Spring Harbor Laboratory Press, 2005; P271 Fig.6)

pared with what we have done on macrolide binding is simply not correct because of the lower resolution of their crystal diffraction. We have been able to find a large number of different antibiotics that bind, and I am not going to go through them all for want of time. We have looked at lots of them. I just want to point out that they bind the different but adjacent sites (Figure 10). So here is place where peptide bond formation occurs. We can bind anisomycin, puromycin, virginiamycin, blastociden, chloramphenical-actually, Ada Yonath's group sees it correctly up here in the PTC-and carbomycin. We also have many other antibiotic complexes. So the point is that these different chemical types are binding to different but close places, so what you can do to create a new drug is tie them together.

I am going to talk now for the last five minutes about some work that comes entirely from Rib-X Pharmaceuticals but was enabled initially by the structures of these antibiotic complexes. This is the company that I and some of my colleagues started about five years ago. They have been able to develop a strategy that seems to work in making potential antibiotics. What they are do- 
ing is taking the antibiotics that bind nearby but to different sites, and chemically tying a part of one to a part of another; so you can take a macrolide and tie it to something that is nearby to each other or you can take one of these and tie it to another one. That is what they are doing. I am going to give you one example. The example is taking sparsomycin which actually does not distinguish between eukaryotes and eubacteria, and linezolid which is a relatively new antibiotic, very effective, and that is shown right here. They partly overlap at this position. So what is done is to chemically bridge these two molecules, and then modify them a lot. Of course, one has to make some modifications so that you do not have the properties of sparsomycin, namely that of working against eukaryotes as well as prokaryotes. This slide shows that you can make a compound that is selective against eubacteria and discriminates against eukaryotes, and is beginning to get some compounds with good binding constants. Then what is done is use a combination of computational chemistry - that is, to decide what would fit, what seems to have the right properties, chemical properties, and synthesize a bunch of additional compounds. They go through cycles of compounds-in this case, showing a couple of hundred ones-and then do some microbiology to see how the inhibition or translation is going for prokaryotes versus eukaryotes. Now at the end of the cycles of design and synthesis they get a compound that exhibits very nice selectivity between eukaryotes and eubacteria. Then you look at their effectiveness against some tough strains, and you look at the minimum inhibitory concentrations in micrograms per mil, (if it is below four it is okay). Thus, 0.25 is excellent, two is fine, so they are getting compounds that are effective against two strains that are resistant to other antibiotics. That is just an example.

Then you can look at two compounds. The affect of the Rib-X compound against various kinds of staphylococcus pneumonii, haemophilus influenza, and a couple of vancomycin- and linezolid-resistant strains is compared with that of azithromycin, a macrolide, sold by Pfizer for quite a bit of money. You can see big MIC numbers like 128 , in the case of azithromycin, while all the numbers for the Rib-X compound are below one, a big difference. They have been able to make some compounds that at least in the microbiology assays are very good. They have now taken one compound into phase one clinical trials. I have been traveling recently, so I have not paid attention to what is happening in the last few weeks, but the last I heard they were about three-quarters of the way through phase one and I hope it is going to work. In phase one you are checking for toxicity, bad side effects. They hope to get this compound into phase two shortly. They have a different category of potential antibiotic made by combining a macrolide with another functional group. They like to call it azithromycin-plus, because it is taking azithromycin and trying to make it even better. That they hoped to put in phase one shortly. So the structures I would say are proving to be useful to design new antibiotics.

I want to do two more things before I stop. One is I want to mention people whom I have not mentioned already who have worked on this structure. These include Dan Klein, in my lab, who refined the structure, Betty Freeborn and Larysa Vasylenko, technicians in Peter Moore's lab who made the 50S ribosome for us, and Kevin Wong in Scott Strobel's lab who made most of the substrate analogue compounds that we used.

Finally, without going into any detail-what are we doing now? Why do we not have any new structures to show right away? That is because we are trying to grow crystals of the ribosome captured in all the states. We are trying to obtain crystals of 70 S ribosomes with EF-G that diffract to higher than $7 \AA$ resolution. We have recently gotten some crystals of $70 \mathrm{~S}$ complexed with EF-G. I do not know to how high resolution it is going to diffract ultimately. We want $70 \mathrm{~S}$ with $\mathrm{EF}-\mathrm{Tu}$, captured delivering the aa-tRNA and ask: how does the ribosome tell the difference between the correct tRNA and the wrong one, so that it knows to hydrolyze the GTP, and how does it know when to hydrolyze the GTP, after the codon has been correctly recognized by the tRNA anticodon $70 \AA$ away? How do you do that? We have to have some structures of ribosome complexes with factors. We want to look at the release factor complex. Venki Ramakrishnan has one at $5 \AA$ resolution, but we still do not know what the factor is doing to stimulate the hydrolysis of that peptide bond; that process is chemistry, we do not know its structural basis yet. Then we want to understand how the ribosome makes polypeptides go through the translocon. A structure of the translocon has been determined, and there are some reasonable electron microscope (EM) pictures of the ribosome bound to the translocon, but I want to know how does the translocon know when to open up so that a membrane protein can go into the membrane? How does any polypeptide sequence to go through this channel without its leaking protons? How does that happen? All of us can make up nice stories, but I want to know the answers. So we are working on all of those questions. The other problem we want to work on is the structure of eukaryotic ribosomes, because eukaryotic protein synthesis is different from prokaryotes in the initiation phase. Particularly the small ribosomal subunit differences are of great interest, and we want to ultimately get structures of the small subunit with initiation factors to understand how this process happens.

Last year, two really good people in the lab got some crystals, and I labeled this slide as "crystals of yeast $40 \mathrm{~S}$ ribosomal subunit". They had obtained crystals of material that sediments 40 S. Some of them diffracted very 
poorly and some of them diffracted to about $3.8 \AA$ resolution. After a while, Ivan Lomakin, for reasons I do not know, ran an SDS gel of one of these crystals and got two bands, one at 210,000 molecular weight and the other one at 220,000 molecular weight. So they solved the structure, and it does not look much like ribosome. It turns out it is yeast fatty acid synthase, which sediments at $40 \mathrm{~S}$. So what they were doing was purifying their $40 \mathrm{~S}$ ribosomal subunit by crystallizing yeast fatty acid sythase which is a hetero hexamer of two and a half million molecular weight, and I have to say, it is a very fascinating structure. It has eight different active sites in it and all the chemistry of fatty acid synthesis is happening in the inside of this large assembly. Although it can be a great distraction from our ribosome work, we shall actually try to understand how this magnificent assembly works.

So science is full of surprises, but I think I shall stop here, and say thank you once again very much for my invitation to come.

Chairman (Prof. Yoshikazu Nakamura, Institute for Medical Science, University of Tokyo): Thank you very much, Tom. An extremely wonderful lecture on ribosome. It is a really attractive and great achievement of structure solutions. So probably he will accept questions or discussion. It is open to questions. Yes, Mr. Yokoyama, dir.

Prof. Shigeyuki Yokoyama (University of Tokyo, RIKEN Genome Research Center): [Inaudible] necessary, because of the rigidity of the ribosome structure?

Prof. Steitz: How much have we considered the possibility that new drugs will change the structure? In the ideal world, of course, you would like to design a drug that does not do that, because of course as you know, changing the structure, unless the two structures are very close in energy, is going to cost energy and that will reduce the binding constants of the compound. So I think nature, as far as I can tell, seems to have evolved inhibitors that produce not many changes, and so the antibiotics that we have bound by and large do not change the structure very much at all. The only ones that change are bases that we can sometimes see a couple of positions even in the absence of the inhibitors so that the energy costs of moving it is very small. So I think our goal is to target the structure that we see so that we do not have to pay the energy penalty of changing the structure.

Chairman: Any other questions? Yes.

Unidentified speaker: I liked your video very much, and that was an assembly of snapshots. I am wondering what is the real speed relative to this. Do you have a comment on that?

Prof. Steitz: It is faster. I do not know; it is many orders of magnitude, though. I think it is about 50-100 peptide bonds form per second, so it is much faster than the movie which forms one peptide bond in about 3 minutes. Chairman: Any other questions? So Tom, you have now the new compound antibiotics candidate, and what would be the frequency of the resistant one appearing against it?

Prof. Steitz: Well, as I like to say in the United States, where as you know intelligent design is one of those things that comes up in discussion of the origin of the universe, I always say that evolution trumps intelligent design, and that is true with antibiotics and I think it is also true with how we arose. It is clear that the ribosome will continue to evolve, and will continue to get around those pesky antibiotics. I suppose if you are in the pharmaceutical business, you can consider this a plus, because you know you will always have a job to do, but I do not see any way in which one can come up with an antibiotic in which you are never going to have to deal with this problem. I think you just have to have continued creativity at a faster rate than the bugs.

Chairman: Yes, definitely we need new compounds. Any other questions? Yes.

Prof. Hideyuki Okano (Department of Physiology, Keio University School of Medicine): You made comments about the future direction. I was very interested in that case. So is the general idea that as regards eukaryotic ribosome there is some membrane-bound and pre-ribosome, and it is likely that membrane-bound is forming a complex translocon, as you call it. Is it some induced fitting structural differences between the membranebound and pre-ribosomes? Because they are very different in the function. So in the case of membrane-bound, the peptide should go into the endoplasmic reticulum. Could you tell the structural differences between these two ribosomes in eukaryote? Can you predict it from the present situation?

Prof. Steitz: Actually, both prokaryotic ribosomes and eukaryotic ribosomes will secrete through the translocon, so there are some similarities there for both eukaryotes and prokaryotes. There are some differences as well, for sure. But the translocon itself is what is embedded in the membrane, and the ribosome sits on top, and so probably the best structural work that is out there are the moment is from Joachim Frank's lab, published last year. At about $12 \AA$ resolution. They can actually fit two translocon molecules into the translocon density and they can fit a ribosome onto it, and they can actually see the polypeptide going down the tunnel and going through the translocon. The problem is, again, detail. You cannot see what the interactions are in detail. The translocon is interacting with the ribosome through a couple of RNA loops that are coming down. So the ribosome itself stays pretty clear of the membranes, certainly the non-polar part of the membrane. So I do not think that the membrane itself is going to alter the ribosome structure. As far as I know, there is no evidence so far from the EM 
that there is a significant difference in the ribosome structure that results from the interaction with the translocon.

Chairman: Any other questions? Yes.

Unidentified speaker: How did you carry out the genetic study of the large ribosome; for example, how do you generate mutations?

Prof. Steitz: That is a longish story. It gets particularly long because the generation time of Haloarcula marismortui is extremely slow; it is half a day or so. Basically, what they do is insert pieces of DNA selectively into the haloarcula marismortui genome. Basically it is the same approach to making mutations as is used in other systems. There are some complications having to do with the fact that there are three copies of the 23S rRNA in the chromosome. We were told when we started this there were only two copies, but we discovered in fact that there were three. So the reason why, for example, on one of the slides I said it was only $33 \%$ mutant 50 S subunit is because it was easy or easier to replace one copy initially, but we then got rid of a second gene copy and that took a little bit more work. It is more or less standard genetics except that the haloarcula marismortui system itself is a little more of a challenge.

Chairman: Okay. One last question. Yes.

Mr.Ryo Miyakawa (An undergraduate student, Keio University School of Medicine): You talked about the antibiotics designed to block the active site; however, I thought you said the active site is very well conserved, which means that in order to gain the resistance against the antibiotics it takes only one or two mutations to get the resistance.

\section{Prof. Steitz: Yes.}

Mr. Miyakawa: Why is there no antibiotics targeting the not so well conserved areas of the ribosome? That way, it will take a few more mutations, and therefore a lot more time to gain resistance against the antibiotics.

Prof. Steitz: Those are both very good questions. I had tried to indicate the answers, but I will be a little clearer about it. The catalytic part of the peptidyl transfer center is indeed very similar or essentially identical. Consequently some of those antibiotics, like blastocydin, are antibiotics in the sense that they will inhibit protein synthesis, they are just as effective against mice as they are against man as they are against bacteria, because that part is absolutely conserved, and indeed that is a bad target. In fact, it is not a target. Now, it turns out that a little bit further down the tunnel in the region where I showed the macrolides and some of those other antibiotics binding, and in the site where linezolid binds, there are some differences. The differences are sometimes one or two nucleotides away from the surface, but they change the shape of the surface enough so that there is some specificity difference. Now as to the question of why not target some place that is completely different in the two kingdoms: the reason they are structurally different is that it does not matter so much to function what the nucleotide is there. So that means you can bind whatever you want in that region, and it is not going to inhibit function. Another binding site that is a great target if you want to make a rat poison but is not so good if you want to kill bacteria is the $\mathrm{E}$ site, because it turns out the structure of the E site is very different in eubacteria and eukaryotes. There is a protein in eukaryotes that is in the region and it is interacting with the CCA end of tRNA, and there are antibiotics-we are about to publish a paper showing several such antibiotics-that will bind specifically to Haloarcula marismortui E site because of course it looks like a eukaryote, but will not bind to eubacteria. That is nice except that you would actually like to do it the other way around. It is not so clear to me that that is going to be easy to do, but it is a great place in terms of difference. It is a big difference, and obviously the E site is functionally important. So we are thinking about that but it is not so obvious what to do.

Chairman: Okay, thank you very much, Tom.

Finally, I would like to make some words before closing this symposium. First of all, it is a really exciting and impressive one-day symposium, and every speaker gave a very excellent talk. Then we learned lots about the RNA from two different aspects. One aspect is about basic research of RNA science. The other additional aspect is applied science, and particularly drug design and therapeutic development going well. So it seems to me that now we have an excellent high-tech car made of RNA, so this RNA car has an excellent two wheels. One wheel is the basic research wheel, and the other wheel is an applied science wheel. We have now lots of gasoline and we can drive this wheel and rotate efficiently and coordinatedly. Hence this RNA car moves very elegantly and rapidly and is going forwards. So it seems this is a kind of stage of RNA science which we are doing in every research effort. This is something like kintaro ame in Japanese. Kintaro ame is a candy bar in which the same face -Kintaro's face, this is a superman-his face is printed inside the bar. Therefore one slice at any position, there appears the same face-Kintaro's face. This is RNA. Now this is it. Yesterday, Tom said that he had visited Japan more than 20 years ago, and at that time I also joined the symposium seminar. It was Oji seminar held in Hokkaido, in Tomokomai. At that time, Joan was invited, and she was working on splicing machinery, and she discovered a new small RNA which was important for the splicing system, so she was really a big star at that moment. Tom accompanied Joan. Somehow I was really impressed about you and we met for the first time there. Next time, it was a very impressive occasion for me in 1999; we all met in the small Greek island Spetses. Every three or four years, one advanced lecture course is organized in the small island of Spetses. Marianne Grun- 
berg-Manago, my mentor, organized this lecture course. On that occasion, Tom gave a five angstrom resolution structure. The paper was published in Nature that year, but really for me, it was the first time to listen to the angstrom atomic resolution. It was so impressive; it was one of my most impressive lectures ever. So, many stories and I am very happy to say that we shared some aspects together in translational control fields. Finally, I would like to express my big congratulations to Tom Steitz for receiving the year 2006 Keio Medical Science Prize this year. I also would like to sincerely respect the committee members in Keio University for their decision of this prize, before Tom gets a Nobel prize, because I am very confident that the Nobel prize will go to Tom Steitz. Hence, this is a very, very excellent decision that Keio University made. Finally, I would like to thank all the speakers and audience for being here and stimulating this discussion, and making it a very joyful meeting here, and finally next door everyone is invited to the get-together party with very nice food and very nice drinks ready and everybody is invited to next door. So we would like to close this symposium. Thank you very much.

\section{References}

1. Ban, N., Freeborn, B., Nissen, P., Penczek, P., Grassucci, P.A., Sweet, R., Frank, J., Moore, P.B. and Steitz, T.A: A $9 \AA$ A resolution $\mathrm{X}$-ray crystallographic map of the large ribosomal subunit. Cell 1998; 93: 4848-4857

2. Ban, N., Nissen, P., Hansen, J., Moore, P.B. and Steitz, T.A: The complete atomic structure of the large ribosomal subunit at $2.4 \AA$ resolution. Science 2000; 289: 905-920

3. Hansen, J. L., Schmeing, T. M., Moore, P. B. and Steitz, T. A: Structural insights into peptide bond formation. Proc. Natl. Acad. Sci. USA 2002; 99: 11670-11675.

4. Schmeing, T. M., Huang, K. S., Strobel, S.A. and Steitz, T.A: An induced-fit mechanism to promote peptide bond formation and exclude hydrolysis of peptidyl-tRNA. Nature 2005a; 438: 520_524
5. Youngman, E. M., Brunelle, J. L., Kochaniak, A. B. and Green, $\mathrm{R}$ : The active site of the ribosome is composed of two layers of conserved nucleotides with distinct roles in peptide bond formation and peptide release. Cell 2004; 117: 589-599

6. Weinger, J. S., Parnell, K. M., Dorner, S., Green, R. and Strobel, S. A: Substrate-assisted catalysis of peptide bond formation by the ribosome. Nature Struc. Biol. 2004;11: 1101-1106

7. Dorner, S., Panuschka, F., Schmid, W. and Barta, A: Mononucleoditde derivatives as ribosomal P-site substrates reveal an important contribution of the 2'-OH activity. Nucl. Acids Res. 2003; 31: $6536-6542$

8. Schmeing, T. M., Huang, K. S., Kitchen, D. E., Strobel, S. A. and Steitz, T. A: Structural insights into the mechanism of the peptidyl transferase reaction suggest oxyanion transition state stabilization by water and a substrate proton shuttle. Molec. Cell 2005b; 20: 437-448

9. Tu, D., Blaha, G., Moore, P.B. and Steitz, T.A: Structures of $\mathrm{MLS}_{\mathrm{B}} \mathrm{K}$ antibiotics bound to mutated large ribosomal subunits provide a structural explanation for resistance. Cell 2005; 121: $257-270$

10. Milligan, R.A. and Unwin, P.N: In vitro crystallization of ribosomes from chick embryos. J. Cell Biol. 1982; 95: 648-653

11. Wimberly, B.T., Brodersen, D.E., Clemons, W.M., Morgan-Warren, R.J., Carter, A.P., Vonshein, C., Hartsch, T., and Ramakrishnan, V: Structure of the 30S ribosomal subunit. Nature 2000; 407: 327-339

12. Yusupov, M. M., Yusupova, G. Z., Baucom, A., Lieberman, K., Earnest, T. N., Cate, J. H. D. and Noller, H. F: Crystal structure of the ribosome at $5.5 \AA$ resolution. Science 2001; 292: 883-896

13. Nissen, P., Ippolito, J. A., Ban, N., Moore, P. B. and Steitz, T. A: RNA tertiary interactions in the large ribosomal subunit: the A-minor motif. Proc. Natl. Acad. Sci. USA 2001; 98: 4899-4903

14. Schmeing, T.M., Seila, A.C., Hansen, J.L., Freeborn, B., Soukup, S.K., et al.; Nature Struct. Biol. 2002; 9: 225-230

15. Hansen, J.L., Ippolito, J.A., Ban, N., Nissen, P., Moore, P.B. and Steitz, T.A: The structures of four macrolide antibiotics bound to the large ribosomal subunit. Mol. Cell 2002b; 10: 117-128

16. Hansen, J.L., Moore, P.B. and Steitz, TA: Structures of five antibiotics bound at the peptidyl transferase center of the large ribosomal subunit. J. Mol. Biol. 2003; 330: 1061-1075 